\title{
Erythroid Maturation Pool Count
}

National Cancer Institute

\section{Source}

National Cancer Institute. Erythroid Maturation Pool Count. NCI Thesaurus. Code C135416.

The determination of the amount of erythroid maturation phase cells (polychromatic rubricytes, normochromic rubricytes, and metarubricytes) present in a sample. 